\title{
Consensus for NF clinical trials
}

\author{
Recommendations of the REiNS collaboration (Supplement II)
}

Brigitte C. Widemann, $\mathrm{MD}$

Scott R. Plotkin, MD, $\mathrm{PhD}$

Correspondence to Dr. Widemann:

widemanb@mail.nih.gov

\section{ABSTRACT}

The neurofibromatoses (NFs) can result in substantial morbidity in affected patients. In order to accelerate the development of effective therapies for NF-related tumor and nontumor manifestations, the development of standardized meaningful outcome measures for clinical trials is critical. The Response Evaluation in Neurofibromatosis and Schwannomatosis (REiNS) International Collaboration was established with the goal to develop consensus recommendations for outcome measures and endpoints in future NF trials and provided the first set of recommendations in a Neurology ${ }^{\circledR}$ supplement in 2013 . This second supplement updates on clinical trials that have incorporated the recommended measures and provides new recommendations for (1) standardized and specific cognitive assessment tools for use in NF clinical trials, (2) patient-reported outcome measures including pain and physical functioning, (3) functional outcome measures for airway plexiform neurofibromas, (4) the use of whole-body MRI in NF, and (5) the development of biomarkers in NF and guidelines for collection of biospecimens and establishment of biobanks for neurofibromatosis 1 (NF1), neurofibromatosis 2 (NF2), and schwannomatosis. Through engagement of the NF research community, regulatory agencies, NF advocacy groups, industry, and patients with NF, REiNS will provide a framework for comprehensive review of these recommendations, continue development of outcome measures relevant to patients, and compare results of trials, which use identical outcome measures. Neurology ${ }^{\circledR}$ 2016;87 (Suppl 1):S1-S3

\section{GLOSSARY}

NF = neurofibromatosis; NF1 = neurofibromatosis 1; NF2 = neurofibromatosis 2; NRS-11 = Numeric Rating Scale-11; PRO $=$ patient-reported outcomes; REiNS $=$ Response Evaluation in Neurofibromatosis and Schwannomatosis.

The NFs-including NF1, NF2, and schwannomatosis-are genetic tumor-predisposition syndromes characterized by the development of multiple tumor manifestations, in particular nerve sheath tumors, for most of which no standard treatment options other than surgery exist. ${ }^{1-3}$ In addition, patients with NF can develop multiple nontumor manifestations, affecting many organ systems, which can result in substantial morbidity and reduced quality of life. There is an unmet need for effective medical therapies for NF-related manifestations. Differences between NF-related tumors and nonsyndromic solid cancers have limited the applicability of clinical trial designs used in oncology trials to the development of trials for NF. ${ }^{4}$

The REiNS International Collaboration was established in 2011 with the primary goal to achieve consensus within the NF community for outcome measures and endpoints for future NF trials. A description of the REiNS International Collaboration and its 7 working groups and initial recommendations of 4 working groups were published previously in Neurology. ${ }^{5-10}$ In brief, the imaging working group provided recommendations for the use of MRI with volumetric analysis to evaluate response in clinical trials targeting histologically benign nerve sheath tumors such as plexiform neurofibromas and vestibular schwannomas. ${ }^{5}$ The patientreported outcomes (PRO) working group described their approach for the objective selection of PRO measures in NF trials, and they recommended the use of Numeric Rating Scale-11 (NRS11) as a measure of pain intensity for manifestations associated with pain. ${ }^{10}$ The visual outcomes working group recommended the use of visual acuity as primary endpoint in clinical trials for

From the Pediatric Oncology Branch (B.C.W.), National Cancer Institute, Bethesda, MD; and Neurology Department and Cancer Center (S.R.P.), Massachusetts General Hospital, Boston.

Go to Neurology.org for full disclosures. Funding information and disclosures deemed relevant by the authors, if any, are provided at the end of the article. 
optic pathway gliomas as opposed to measurement of tumor size. ${ }^{6}$ Finally, the functional outcomes working group provided recommendations for hearing response measured by word recognition as primary endpoint in trials for NF2-related vestibular schwannomas. ${ }^{7}$ Since the publication of these recommendations in 2013, a number of ongoing clinical trials have incorporated these outcome measures.

Incorporation of REiNS-recommended outcome measures into ongoing clinical trials. Imaging response in clinical trials. The phase II trials of the MEK inhibitor PD0325901 (NCT02096471), the receptor tyrosine kinase inhibitor cabozantinib (NCT02101736), and the MEK inhibitor selumetinib (NCT01362803) ${ }^{11}$ directed at NF1-related inoperable plexiform neurofibromas are using volumetric MRI analysis and the response criteria provided by REiNS to define the activity of the agents under investigation. Several clinical trials directed at NF2-related vestibular schwannomas have also used volumetric MRI analysis to sensitively monitor changes in tumor size as a trial endpoint (NCT01207687, NCT01767792, NCT01419639, NCT00973739).

Patient-reported outcomes. Pain is a common and debilitating manifestation in NF-related tumors that is often difficult to treat. The evaluation of pain intensity using the NRS-11 scale has been incorporated into 5 ongoing trials with targeted investigational therapies for NF-related plexiform neurofibromas (NCT02390752, NCT02096471, NCT01362803, NCT02407405, NCT02101736) and in one treatment trial targeting malignant peripheral nerve sheath tumors (NCT02008877). ${ }^{12}$

Subsequent recommendations by the PRO working group for measures of physical functioning using the Patient-Reported Outcome Measurement Information System scale (provided in this supplement) have already been incorporated in (1) 2 treatment trials for NF1-related plexiform neurofibromas (NCT01362803, NCT02407405); (2) behavioral intervention trials of acceptance and commitment therapy (NCT01633008, NCT02471339) and resilience training (NCT02387177); and (3) assessment of psychosocial outcomes (NCT02435628). The PRO group has also initiated trials to validate the English version of the Pain Interference Index and the Pain Rating Scale in NF1 (NCT01639950), the adolescent PROMIS computerized adaptive test in NF1 and NF2, to explore modifications to the NRS-11, Pain Interference Index, and PROMIS-Physical Functioning, and to make these measures available electronically (NCT02544022).

Visual outcomes. The development and progression of orbital plexiform neurofibromas in NF1 results in vision loss in the affected eye in many patients. In a phase II clinical trial of the MEK inhibitor selumetinib (NCT01362803) for inoperable NF1 plexiform neurofibromas, vision measurements as recommended by the visual outcomes group have been incorporated as a measure of clinically meaningful improvement. These measures are also used in an ongoing longitudinal natural history study of NF1 optic pathway tumors.

Functional outcomes. The measurement of word recognition as a direct measure of clinical benefit in patients with NF2 and vestibular schwannomas resulting in hearing loss has been incorporated as a primary trial endpoint into 2 multi-institutional phase II trials of bevacizumab (NCT01207687, NCT01767792), and as a secondary trial endpoint into phase II trials of everolimus $^{13}$ (NCT01419639), lapatinib ${ }^{14}$ (NCT00973739), and axitinib (NCT02129647). Recommendations for outcome measures for plexiform neurofibromas affecting the airway in NF1, which are provided in this second supplement, have been incorporated in the ongoing phase II trial of selumetinib for NF1-related plexiform neurofibromas for the subset of patients with airway tumors (NCT01362803). These recommendations will also be incorporated as the primary endpoint in a planned phase II study of imatinib for NF1-related airway plexiform neurofibromas.

The incorporation of REiNS recommendations into multiple trials reflects broad acceptance of REiNS recommendations by the NF research community and will allow for meaningful comparisons of results across trials.

Additional REiNS recommendations provided in this supplement. This second supplement presents recommendations of 4 REiNS working groups developed since 2013.

Neurocognitive working group. NF1 is associated with neurocognitive deficits, which can affect everyday functioning of children, adolescents, and adults with this disease. The working group describes its approach to the identification of standardized and specific cognitive assessment tools for use in NF clinical trials and provides recommendations for measurement of attention as a domain of high priority for clinical trials.

Patient-reported outcomes. The PRO group continues to address the complex subjective nature of pain by addressing multiple domains of importance to patients. The group provides recommendations for assessment of pain intensity, pain interference, and physical functioning in clinical trials for NF.

Functional outcomes. The functional outcomes working group proposes outcome measures for sleep and pulmonary function for the subgroup of patients with NF1-related plexiform neurofibromas affecting the airway. 
Whole-body MRI. Given that nerve sheath tumors can affect multiple body regions in patients with NF, whole-body MRI has been used by several investigators to assess total body tumor burden. The whole-body MRI working group presents a systematic review of the use of whole-body MRI and highlights areas in need of further study.

Biomarker working group. There is a great need for the development of validated biomarkers in NF. The biomarker working group provides a review of existing biomarkers in NF and proposes standard operating procedures and a minimal clinical dataset for future biomarker studies in this supplement.

Future goals and plans. The goal of REiNS is to continue the development of outcome measures of NF manifestations in clinical trials. Currently, groups are working to establish consensus recommendations for outcome measures for trials addressing bony manifestations in NF, to validate and compare 2 methods of volumetric MRI analysis of plexiform neurofibromas, and to develop guidelines for the assessment of malignant transformation of NF1-related tumors. Through biannual meetings open to the NF research community and with participation by the Food and Drug Administration, European Medicines Agency, the Cancer Therapy Evaluation Program, NF advocacy groups, and industry, REiNS will seek feedback for ongoing recommendations and guidance for new initiatives. Importantly, REiNS is actively exploring how best to involve patients and caregivers into this process of developing endpoints most relevant to patients. Over the next few years, results of trials using REiNS outcome measures will be published.

NF1, NF2, and schwannomatosis are rare conditions and novel approaches to drug development are needed. Although the number of clinical trials for NF has increased in recent years, large, randomized studies are not currently feasible. However, by using similar eligibility criteria and identical outcome measures, the NF community hopes to promote meaningful comparisons of agents across clinical trials.

\section{AUTHOR CONTRIBUTIONS}

Brigitte C. Widemann: drafting/revising the manuscript, study concept or design, accepts responsibility for conduct of research and final approval. Scott R. Plotkin: drafting/revising the manuscript, study concept or design, accepts responsibility for conduct of research and final approval, study supervision, obtaining funding.

\section{ACKNOWLEDGMENT}

The authors acknowledge the support of the Children's Tumor Foundation for the REiNS International Collaboration and this supplement.

\section{STUDY FUNDING}

No targeted funding reported.

\section{DISCLOSURE}

B. Widemann: research support through NCI CCR Intramural Research Program. S. Plotkin: research support from the Children's Tumor Foundation, NIH, Department of Defense Neurofibromatosis Clinical Trials Consortium. Go to Neurology.org for full disclosures.

Received November 20, 2015. Accepted in final form May 20, 2016.

\section{REFERENCES}

1. Evans DG, Ramsden RT, Gokhale C, Bowers N, Huson SM, Wallace A. Should NF2 mutation screening be undertaken in patients with an apparently isolated vestibular schwannoma? Clin Genet 2007;71:354-358.

2. Ferner RE. Neurofibromatosis 1 and neurofibromatosis 2 : a twenty first century perspective. Lancet Neurol 2007;6: 340-351.

3. Merker VL, Esparza S, Smith MJ, StemmerRachamimov A, Plotkin SR. Clinical features of schwannomatosis: a retrospective analysis of 87 patients. Oncologist 2012;17:1317-1322.

4. Gutmann DH, Blakeley JO, Korf BR, Packer RJ. Optimizing biologically targeted clinical trials for neurofibromatosis. Expert Opin Investig Drugs 2013;22:443-462.

5. Dombi E, Ardern-Holmes SL, Babovic-Vuksanovic D, et al. Recommendations for imaging tumor response in neurofibromatosis clinical trials. Neurology 2013;81:S33-S40.

6. Fisher MJ, Avery RA, Allen JC, et al. Functional outcome measures for NF1-associated optic pathway glioma clinical trials. Neurology 2013;81:S15-S24.

7. Plotkin SR, Ardern-Holmes SL, Barker FG II, et al. Hearing and facial function outcomes for neurofibromatosis 2 clinical trials. Neurology 2013;81:S25-S32.

8. Plotkin SR, Blakeley JO, Dombi E, et al. Achieving consensus for clinical trials: the REiNS International Collaboration. Neurology 2013;81:S1-S5.

9. Widemann BC, Blakeley JO, Dombi E, et al. Conclusions and future directions for the REiNS international collaboration. Neurology 2013;81:S41-S44.

10. Wolters PL, Martin S, Merker VL, et al. Patient-reported outcomes in neurofibromatosis and schwannomatosis clinical trials. Neurology 2013;81:S6-S14.

11. Widemann B, Marcus L, Fisher M, et al. Phase I study of the MEK1/2 inhibitor selumetinib (AZD6244) hydrogen sulfate in children and young adults with neurofibromatosis type 1 (NF1) and inoperable plexiform neurofibromas (PNs). J Clin Oncol 2014;32:5s. Abstract.

12. Kim A, Reinke D, Cichowski K, et al. SARC023: phase I/II trial of ganetespib in combination with sirolimus for refractory sarcomas and malignant peripheral nerve sheath tumors (MPNST). J Clin Oncol 2014;32:5s. Abstract.

13. Karajannis MA, Legault G, Hagiwara M, et al. Phase II study of everolimus in children and adults with neurofibromatosis type 2 and progressive vestibular schwannomas. Neuro Oncol 2014;16:292-297.

14. Karajannis MA, Legault G, Hagiwara M, et al. Phase II trial of lapatinib in adult and pediatric patients with neurofibromatosis type 2 and progressive vestibular schwannomas. Neuro Oncol 2012;14:1163-1170. 


\section{Neurology}

\section{Consensus for NF clinical trials: Recommendations of the REiNS collaboration (Supplement II) \\ Brigitte C. Widemann and Scott R. Plotkin \\ Neurology 2016;87;S1-S3 \\ DOI 10.1212/WNL.0000000000002930}

This information is current as of August 15, 2016

\section{Updated Information \& Services}

References

Citations

Subspecialty Collections

Permissions \& Licensing

Reprints including high resolution figures, can be found at: http://n.neurology.org/content/87/7_Supplement_1/S1.full

This article cites 14 articles, 1 of which you can access for free at: http://n.neurology.org/content/87/7_Supplement_1/S1.full\#ref-list-1

This article has been cited by 1 HighWire-hosted articles: http://n.neurology.org/content/87/7_Supplement_1/S1.full\#\#otherarticl es

This article, along with others on similar topics, appears in the following collection(s):

Neurofibromatosis

http://n.neurology.org/cgi/collection/neurofibromatosis

Information about reproducing this article in parts (figures,tables) or in its entirety can be found online at:

http://www.neurology.org/about/about_the_journal\#permissions

Information about ordering reprints can be found online:

http://n.neurology.org/subscribers/advertise

Neurology ${ }^{\circledR}$ is the official journal of the American Academy of Neurology. Published continuously since 1951, it is now a weekly with 48 issues per year. Copyright (O 2016 American Academy of Neurology. All rights reserved. Print ISSN: 0028-3878. Online ISSN: 1526-632X.

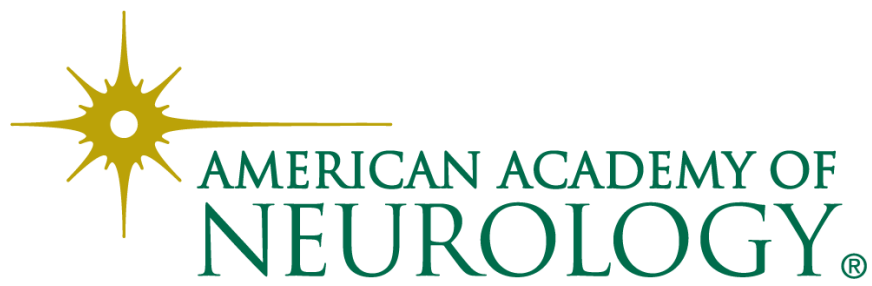

